\title{
Computacional Characterization of a Complete Palladium-Catalyzed Cross- coupling Process: The Associative Transmetalation in the Stille Reaction
}

Rosana Álvarez, ${ }^{*}$ Olalla Nieto-Faza, Carlos Silva-López, and Angel R. de Lera*

Departamento de Química Orgánica, Facultad de Química, Universidade de Vigo,

Lagoas-Marcosende s/n, 36310 Vigo, Spain

$\begin{array}{ll}\text { S1 } & \text { Table of contents } \\ \text { S2 } & \text { Computacional Details } \\ \text { S3 } & \left.\text { Reaction Energy Profile in the gas phase and in solvent (PCM: THF and } \mathrm{CH}_{3} \mathrm{CN}\right) \\ \text { S4-S5 } & \begin{array}{l}\text { Absolute and relative energies }(\mathrm{Kcal} / \mathrm{mol}) \text { of the species along the mechanistic } \\ \text { pathway }\end{array}\end{array}$

S6-S23 Cartesian Coordinates of the computed structures 


\section{Computacional Details:}

All computations in this study have been performed using the Gaussian03 suite of programs(cita). To include electron correlation at a reasonable computational cost, density functional theory (DFT) was used(cita). The Becke three-parameter exchange functional(cita) and the nonlocal correlation functional of Lee, Yang, and Parr(cita) (B3LYP) with the 6-31G* set basis for $\mathrm{C}, \mathrm{H}$ and $\mathrm{P}$, in conjunction with the Stuttgart/Dresden relativistic effective core potentials(cita) for $\mathrm{Pd}, \mathrm{Sn}$ and $\mathrm{Br}$ were used to compute the geometries, energies, and vibrational frequencies of the minima and transition structures. All the stationary points were characterized by means of harmonic analysis, and for all transition structures, the normal mode associated to the imaginary frequency corresponds to the nuclear motion along the reaction coordinate under study.

Single-point solvation calculations (in THF and $\mathrm{CH}_{3} \mathrm{CN}$ ) were performed the optimized geometries of some of the systems under study, using the polarizable continuum model (PCM) (J. Comput Chem 1998, 19, 290, J. Chem. Phys 2002, 117, 43) and a variation of the conductor-like screening model (COSMO employing the parameters provided by Klamt and coworkers) (J. Phys. Chem. A 1998, 102, 5074) as implemented in GAUSSIANO3 (J. Phys. Chem A 1998, 102, 1995) with the UAKS radii (J. Chem. Phys 1997, 107, 3210). 


\begin{tabular}{|c|c|c|c|c|c|c|}
\hline $\begin{array}{l}\text { kcals/mol } \\
\text { FILE }\end{array}$ & & SCF & SCF+zpve & Enthalpy & & ree energy \\
\hline i & & -659008.16 & -658863.91 & -658852.73 & 40.19 & -658892.92 \\
\hline & 1 & -57329.71 & -57303.29 & -57300.37 & 19.67 & -57320.04 \\
\hline TSi-ii & & -716331.11 & -716160.76 & -716146.64 & 47.44 & -716194.08 \\
\hline ii & & -716363.13 & -716190.98 & -716176.98 & 45.76 & -716222.74 \\
\hline TSii-iii & & -872304.83 & -872067.35 & -872048.76 & 56.9 & -872105.66 \\
\hline iii & & -872305.72 & -872067.66 & -872048.76 & 57.94 & -872106.7 \\
\hline TSiii-iv & & -872297.46 & -872060.69 & -872041.7 & 59.44 & -872101.13 \\
\hline iv & & -582961.67 & -582796.38 & -582782.4 & 47.81 & -582830.21 \\
\hline TSiv-v & & -709143.7 & -708883.92 & -708861.76 & 68.15 & -708929.91 \\
\hline $\mathbf{v}$ & & -553208.38 & -553012.97 & -552995.85 & 54.42 & -553050.27 \\
\hline TSv-viii & & -553187.19 & -552992.72 & -552975.75 & 54.09 & -553029.84 \\
\hline viii & & -553191.22 & -552997.21 & -552979.4 & 58.04 & -553037.44 \\
\hline ix & & -467497.61 & -467373.52 & -467363.27 & 38.3 & -467401.57 \\
\hline TS1ix-i & & -467494.86 & -467370.72 & -467361.04 & 36.46 & -467397.5 \\
\hline TS2ix-i & & -467537.62 & -467411.41 & -467402.24 & 35.29 & -467437.54 \\
\hline & 3 & -97884.59 & -97830.93 & -97827.42 & 19.69 & -97847.11 \\
\hline TSii-vi & & -1005702.16 & -1005458.04 & -1005439.23 & 56.4 & -1005495.63 \\
\hline vi & & -1005709.53 & -1005463.61 & -1005444.96 & 55.81 & -1005500.77 \\
\hline TSvi-vii & & -1005707.11 & -1005463.45 & -1005444.38 & 57.19 & -1005501.57 \\
\hline vii & & -716374.02 & -716201.99 & -716187.87 & 46.18 & -716234.04 \\
\hline TSvii-v & & -842547.09 & -842280.2 & -842258.16 & 65.59 & -842323.75 \\
\hline x.DMF & & -155937.59 & -155873.35 & -155869.68 & 21.23 & -155890.91 \\
\hline xiii.Br & & -8453.81 & -8453.81 & -8452.33 & 11.63 & -8463.96 \\
\hline 2.vinilSnMe3 & & -126190.96 & -126096.57 & -126089.14 & 30.92 & -126120.06 \\
\hline xi.PMe3 & & -289341.03 & -289269.85 & -289265.06 & 23.2 & -289288.26 \\
\hline xiv.BrSnMe3 & & -85685.5 & -85616.42 & -85609.33 & 31.04 & -85640.37 \\
\hline
\end{tabular}




\begin{tabular}{|c|c|c|c|c|c|}
\hline \multicolumn{6}{|l|}{ kcal/mol } \\
\hline FILE & & & $\triangle \mathrm{G} P C M(\mathrm{CH} 3 \mathrm{CN})$ & $\Delta \mathrm{G}$ COSMO (THF) & $\Delta \mathrm{G}$ PCM (THF) \\
\hline i & & 6.45 & 9.22 & 2.47 & 5.78 \\
\hline & 1 & 1.7 & 1.75 & -0.11 & 0.13 \\
\hline Ts:i-ii & & 10.48 & 11.2 & 5.96 & 7.61 \\
\hline ii & & 5.6 & 6.55 & 1.04 & 3.3 \\
\hline Ts:ii-iii & & 8.55 & 9.91 & 2.84 & 5.8 \\
\hline iii & & 8.09 & 9.46 & 2.41 & 5.53 \\
\hline Ts:iii-iv & & 11.39 & 12.74 & 5.67 & 8.23 \\
\hline iv & & 6.5 & 1.86 & 0.56 & 4.02 \\
\hline Tsiv-v & & 14.89 & 16.82 & 8.72 & 11.83 \\
\hline $\mathbf{v}$ & & 13.47 & 13.95 & 8.68 & 9.86 \\
\hline Tsv-viii & & 15.08 & 15.57 & 10.36 & 11.38 \\
\hline viii & & 14.71 & 15.55 & 9.78 & 11.3 \\
\hline ix & & 7.66 & 8.29 & 4.22 & 5.3 \\
\hline TS1:ix-3 & & 8.47 & 8.92 & 5 & 5.8 \\
\hline TS2:ix-3 & & 7.48 & 8.51 & 4.09 & 5.46 \\
\hline & 3 & 2.79 & 2.83 & 0.92 & 1.07 \\
\hline ii & & 5.6 & 6.55 & 1.04 & 3.3 \\
\hline TS:ii-vi & & 11.91 & 12.73 & 4.98 & 6.35 \\
\hline vi & & 9.06 & 10.17 & 3.6 & 6.31 \\
\hline TS:vi-vii & & 11.48 & 12.21 & 6.09 & 8.15 \\
\hline vii & & 9.55 & 10.16 & 4.98 & 6.35 \\
\hline TS:vii-v & & 20.91 & 21.48 & 15.09 & 16.36 \\
\hline
\end{tabular}




\section{CARTESIAN COORDINATES}

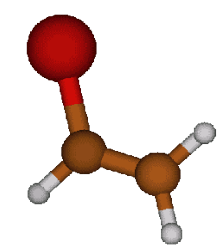

1 SCF Energy: -91.3606360764

$\begin{array}{lrrr}\mathrm{C} & 0.461950 & -1.151305 & 0.000000 \\ \mathrm{C} & -0.446042 & -2.115485 & 0.000000 \\ \mathrm{H} & 1.535189 & -1.293268 & 0.000000 \\ \mathrm{H} & -0.116990 & -3.152045 & 0.000000 \\ \mathrm{H} & -1.513648 & -1.923306 & 0.000000 \\ \mathrm{Br} & 0.000000 & 0.741982 & 0.000000\end{array}$

3 SCF Energy: -155.988895032

$\begin{array}{lrrr}\mathrm{C} & -0.326354 & 0.651739 & 0.000000 \\ \mathrm{C} & 0.326354 & 1.823035 & 0.000000 \\ \mathrm{H} & -1.416772 & 0.644972 & 0.000000 \\ \mathrm{H} & -0.200353 & 2.772554 & 0.000000 \\ \mathrm{H} & 1.413360 & 1.868554 & 0.000000 \\ \mathrm{C} & 0.326354 & -0.651739 & 0.000000 \\ \mathrm{C} & -0.326354 & -1.823035 & 0.000000 \\ \mathrm{H} & 1.416772 & -0.644972 & 0.000000 \\ \mathrm{H} & 0.200353 & -2.772554 & 0.000000 \\ \mathrm{H} & -1.413360 & -1.868554 & 0.000000\end{array}$

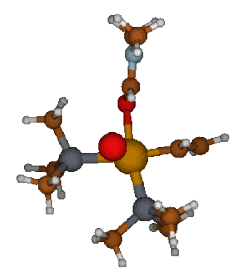

iii

SCF Energy: -1390.10647722

$\begin{array}{lrrr}\mathrm{C} & -0.088276 & -2.374289 & -0.286607 \\ \mathrm{C} & -0.128407 & -3.323081 & -1.226690 \\ \mathrm{Pd} & -0.367099 & -0.378424 & -0.532710 \\ \mathrm{P} & -2.509834 & -0.884117 & 0.012722 \\ \mathrm{P} & -0.422439 & 2.045531 & -0.873354 \\ \mathrm{O} & 1.787831 & -0.320515 & -1.078402\end{array}$




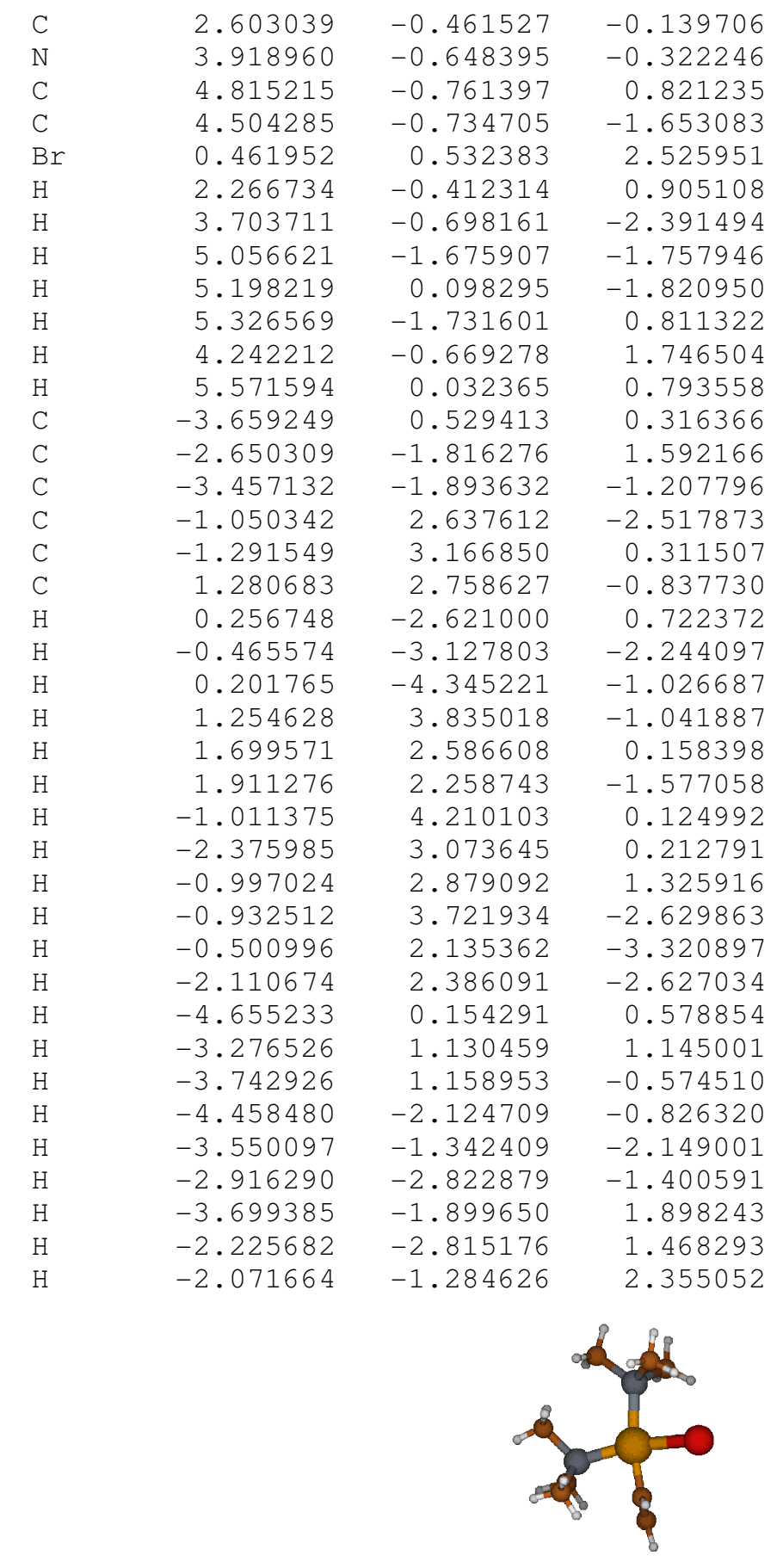

\begin{tabular}{lrrr} 
ii & \multicolumn{3}{c}{} \\
SCF & Energy: -1141.59635766 & \\
C & -2.424437 & 1.581198 & 1.559737 \\
P & -1.279244 & 1.662790 & .111454 \\
C & -2.422343 & 1.860702 & -1.326672 \\
Pd & .118634 & -.341231 & -.033839 \\
Br & -2.097538 & -1.642199 & -.171962 \\
C & -.631446 & 3.396482 &. .288503 \\
P & 2.281795 & .424902 & -.103761 \\
C & 2.590506 & 2.221839 & -.430976 \\
C & .994568 & -2.177652 & -.110449 \\
C & 1.479561 & -2.893486 & .907831 \\
C & 3.262999 & .156011 & 1.435909 \\
C & 3.331907 & -.346917 & -1.412113
\end{tabular}




$\begin{array}{rrrr}\mathrm{H} & -1.460472 & 4.107461 & .381484 \\ \mathrm{H} & -.004426 & 3.478014 & 1.182389 \\ \mathrm{H} & -.031720 & 3.674551 & -.582803 \\ \mathrm{H} & -3.141228 & 2.669612 & -1.152991 \\ \mathrm{H} & -1.842590 & 2.083686 & -2.228634 \\ \mathrm{H} & -2.951714 & .917240 & -1.480928 \\ \mathrm{H} & -3.128559 & 2.421357 & 1.555385 \\ \mathrm{H} & -2.971304 & .636107 & 1.521513 \\ \mathrm{H} & -1.843035 & 1.605610 & 2.487449 \\ \mathrm{H} & 3.667078 & 2.418729 & -.491832 \\ \mathrm{H} & 2.125141 & 2.514515 & -1.377191 \\ \mathrm{H} & 2.165512 & 2.833943 & .367976 \\ \mathrm{H} & 4.295887 & .498001 & 1.304740 \\ \mathrm{H} & 2.805173 & .705156 & 2.264698 \\ \mathrm{H} & 3.261050 & -.908573 & 1.680424 \\ \mathrm{H} & 4.345343 & .069759 & -1.391727 \\ \mathrm{H} & 3.374990 & -1.425874 & -1.249973 \\ \mathrm{H} & 2.887039 & -.162541 & -2.395113 \\ \mathrm{H} & .952513 & -2.622617 & -1.107853 \\ \mathrm{H} & 1.817092 & -3.922746 & .770810 \\ \mathrm{H} & 1.508719 & -2.522260 & 1.931415\end{array}$

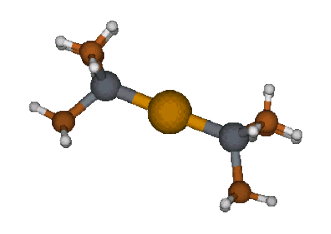

i SCF Energy: -1050.19547690

$\begin{array}{lrrr}\mathrm{C} & 3.151353 & -1.188523 & -1.148935 \\ \mathrm{P} & 2.305252 & 0.000786 & -0.000534 \\ \mathrm{C} & 3.129246 & 1.595981 & -0.474380 \\ \mathrm{C} & 3.174486 & -0.379827 & 1.595770 \\ \mathrm{Pd} & -0.000173 & -0.012954 & 0.011879 \\ \mathrm{P} & -2.305381 & 0.000684 & -0.000531 \\ \mathrm{C} & -3.154784 & -1.175615 & -1.159769 \\ \mathrm{C} & -3.176121 & -0.394836 & 1.591374 \\ \mathrm{C} & -3.123212 & 1.603298 & -0.459585 \\ \mathrm{H} & 4.223116 & 1.515015 & -0.447192 \\ \mathrm{H} & 2.815548 & 1.881274 & -1.483369 \\ \mathrm{H} & 2.811050 & 2.387263 & 0.211498 \\ \mathrm{H} & 4.265360 & -0.351067 & 1.481865 \\ \mathrm{H} & 2.874135 & 0.347584 & 2.356415 \\ \mathrm{H} & 2.876433 & -1.373172 & 1.945128 \\ \mathrm{H} & 4.244074 & -1.108653 & -1.087762 \\ \mathrm{H} & 2.852278 & -2.210961 & -0.897609 \\ \mathrm{H} & 2.834352 & -0.989972 & -2.177374 \\ \mathrm{H} & -4.266858 & -0.352073 & 1.480633 \\ \mathrm{H} & -2.888874 & -1.397164 & 1.924174 \\ \mathrm{H} & -2.865847 & 0.316707 & 2.362892 \\ \mathrm{H} & -4.246997 & -1.082301 & -1.109159 \\ \mathrm{H} & -2.824843 & -0.979022 & -2.184585 \\ \mathrm{H} & -2.871364 & -2.202166 & -0.907549 \\ \mathrm{H} & -4.217323 & 1.525384 & -0.435792 \\ \mathrm{H} & -2.804072 & 2.386343 & 0.235242 \\ \mathrm{H} & -2.806069 & 1.897948 & -1.464791\end{array}$




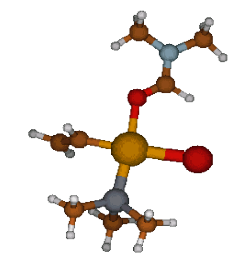

iv

SCF Energy: -929.007769163

$\begin{array}{lrrr}\mathrm{C} & 3.254322 & 0.164108 & 1.617437 \\ \mathrm{P} & 2.557728 & 0.108636 & -0.087282 \\ \mathrm{C} & 3.195475 & -1.477060 & -0.772331 \\ \mathrm{Pd} & 0.314025 & 0.349860 & -0.104255 \\ \mathrm{O} & -1.832161 & 0.827465 & -0.195496 \\ \mathrm{C} & -2.749930 & 0.011214 & 0.015170 \\ \mathrm{~N} & -4.052279 & 0.334923 & -0.028972 \\ \mathrm{C} & -4.489146 & 1.691672 & -0.332502 \\ \mathrm{C} & 3.556917 & 1.359717 & -1.003420 \\ \mathrm{Br} & -0.155230 & -2.230255 & 0.122842 \\ \mathrm{C} & 0.575779 & 2.332936 & -0.160105 \\ \mathrm{C} & 0.816613 & 3.143377 & 0.875021 \\ \mathrm{C} & -5.080410 & -0.667941 & 0.207608 \\ \mathrm{H} & 0.379145 & 2.763656 & -1.147606 \\ \mathrm{H} & 0.799771 & 4.230047 & 0.767124 \\ \mathrm{H} & 1.013743 & 2.773015 & 1.880361 \\ \mathrm{H} & 4.626286 & 1.136419 & -0.914761 \\ \mathrm{H} & 3.275171 & 1.344403 & -2.061011 \\ \mathrm{H} & 3.350602 & 2.355862 & -0.606852 \\ \mathrm{H} & 4.342246 & 0.031735 & 1.601820 \\ \mathrm{H} & 3.013807 & 1.127531 & 2.076012 \\ \mathrm{H} & 2.798610 & -0.630812 & 2.214693 \\ \mathrm{H} & 4.289259 & -1.505534 & -0.701794 \\ \mathrm{H} & 2.754736 & -2.313178 & -0.226187 \\ \mathrm{H} & 2.894137 & -1.567445 & -1.819830 \\ \mathrm{H} & -2.529557 & -1.036660 & 0.248093 \\ \mathrm{H} & -5.082618 & 1.701338 & -1.254751 \\ \mathrm{H} & -5.106584 & 2.079323 & 0.486365 \\ \mathrm{H} & -3.609355 & 2.322132 & -0.457307 \\ \mathrm{H} & -5.724381 & -0.772497 & -0.674103 \\ \mathrm{H} & -4.613045 & -1.632744 & 0.417364 \\ \mathrm{H} & -5.704476 & -0.383073 & 1.063363 \\ & & & \\ & & & \end{array}$

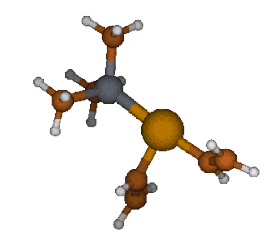

ix

SCF Energy: - 745.004237400

$\begin{array}{lrrr}\mathrm{C} & -2.697441 & -.378093 & -.298073 \\ \mathrm{C} & -3.404699 & -1.340947 & .315560 \\ \mathrm{Pd} & -.703738 & -.271131 & -.108214 \\ \mathrm{C} & -.984308 & 1.575115 & .569836 \\ \mathrm{C} & -1.020127 & 2.696943 & -.153110 \\ \mathrm{P} & 1.705511 & -.221948 & .009560 \\ \mathrm{H} & -3.208746 & .273168 & -1.011647 \\ \mathrm{H} & -1.075344 & 1.609825 & 1.657740 \\ \mathrm{H} & -1.109737 & 3.671311 & .331587\end{array}$




$\begin{array}{rrrr}\mathrm{H} & -.979616 & 2.705307 & -1.239954 \\ \mathrm{C} & 2.659570 & -1.760820 & -.394031 \\ \mathrm{C} & 2.472433 & 1.025211 & -1.118880 \\ \mathrm{C} & 2.409033 & .251190 & 1.653964 \\ \mathrm{H} & -4.454792 & -1.533233 & .079575 \\ \mathrm{H} & -2.975913 & -1.989399 & 1.079858 \\ \mathrm{H} & 3.741091 & -1.598271 & -.319016 \\ \mathrm{H} & 2.374262 & -2.563505 & .293926 \\ \mathrm{H} & 2.421019 & -2.086108 & -1.411936 \\ \mathrm{H} & 3.558802 & 1.084161 & -.985966 \\ \mathrm{H} & 2.251492 & .761477 & -2.158093 \\ \mathrm{H} & 2.026220 & 2.003666 & -.916760 \\ \mathrm{H} & 3.502444 & .322113 & 1.625065 \\ \mathrm{H} & 1.994232 & 1.218603 & 1.953186 \\ \mathrm{H} & 2.117105 & -.489459 & 2.405248\end{array}$

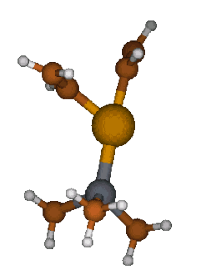

TSix-3

SCF Energy: -744.999849436

$\begin{array}{lrrr}\mathrm{C} & -1.726262 & 1.204651 & .535067 \\ \mathrm{C} & -2.033016 & 2.335216 & -.115384 \\ \mathrm{Pd} & -.556693 & -.296888 & -.151997 \\ \mathrm{C} & -2.543839 & -.582102 & -.379472 \\ \mathrm{C} & -3.256117 & -1.399789 & . .412956 \\ \mathrm{P} & 1.808317 & -.046498 & .029888 \\ \mathrm{H} & -1.897542 & 1.132436 & 1.609876 \\ \mathrm{H} & -4.204639 & -1.830343 & .087581 \\ \mathrm{H} & -2.932643 & -1.667208 & 1.416995 \\ \mathrm{H} & -2.950433 & -.282506 & -1.347613 \\ \mathrm{C} & 2.592836 & 1.078339 & -1.215153 \\ \mathrm{C} & 2.400135 & .683713 & 1.625182 \\ \mathrm{C} & 2.872690 & -1.560589 & -.108847 \\ \mathrm{H} & -2.403327 & 3.207906 & .424141 \\ \mathrm{H} & -1.943689 & 2.438611 & -1.194055 \\ \mathrm{H} & 3.489076 & .812353 & 1.634455 \\ \mathrm{H} & 1.921068 & 1.656263 & 1.774360 \\ \mathrm{H} & 2.108170 & .032955 & 2.455330 \\ \mathrm{H} & 3.937056 & -1.320191 & -.001630 \\ \mathrm{H} & 2.590453 & -2.279345 & .667122 \\ \mathrm{H} & 2.713232 & -2.035522 & -1.082455 \\ \mathrm{H} & 3.670832 & 1.185190 & -1.045615 \\ \mathrm{H} & 2.426464 & .683194 & -2.222317 \\ \mathrm{H} & 2.120497 & 2.063912 & -1.158717\end{array}$

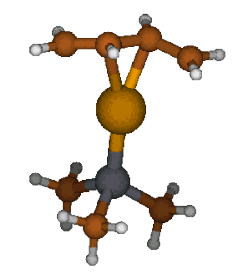

TSix-3'

SCF Energy: -745.068005811

C $\quad-2.367329 \quad-1.845905 \quad-.112740$ 


$\begin{array}{lrrr}\mathrm{C} & -2.662381 & -.610665 & .397948 \\ \mathrm{Pd} & -.509393 & .008482 & .001920 \\ \mathrm{P} & 1.765350 & .003127 & -.002756 \\ \mathrm{C} & -2.660908 & .607773 & -.004974 \\ \mathrm{C} & -2.364246 & 1.842329 & .107733 \\ \mathrm{H} & -2.811819 & .502795 & -1.479332 \\ \mathrm{C} & 2.658459 & 1.577262 & -.426955 \\ \mathrm{C} & 2.595643 & -1.182587 & -1.166683 \\ \mathrm{C} & 2.599796 & -.435201 & 1.597707 \\ \mathrm{H} & -2.816338 & -.505056 & 1.471872 \\ \mathrm{H} & -2.284728 & -2.016107 & -1.182828 \\ \mathrm{H} & -2.326322 & -2.719949 & .528475 \\ \mathrm{H} & -2.319967 & 2.716654 & -.532855 \\ \mathrm{H} & -2.288944 & 2.012713 & 1.178324 \\ \mathrm{H} & 3.689059 & -1.124611 & -1.098003 \\ \mathrm{H} & 2.274196 & -2.202748 & -.934942 \\ \mathrm{H} & 2.290740 & -.959498 & -2.194080 \\ \mathrm{H} & 3.748419 & 1.454445 & -.390454 \\ \mathrm{H} & 2.367953 & 1.901107 & -1.431424 \\ \mathrm{H} & 2.362493 & 2.362200 & .276368 \\ \mathrm{H} & 3.692814 & -.411488 & 1.504663 \\ \mathrm{H} & 2.292880 & .270969 & 2.375664 \\ \mathrm{H} & 2.287181 & -1.436545 & 1.909366 \\ & & & \end{array}$

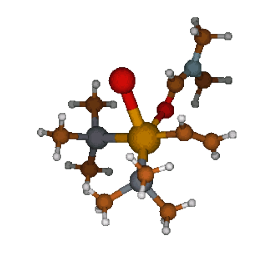

\begin{tabular}{lrrr}
\multicolumn{4}{l}{ TSii-iii } \\
SCF & Energy $:-1390.10506165$ & \\
$\mathrm{C}$ & -3.888105 & 0.150512 & -0.459708 \\
$\mathrm{P}$ & -2.534078 & -1.090767 & -0.219144 \\
$\mathrm{C}$ & -2.953282 & -2.343252 & -1.510914 \\
$\mathrm{Pd}$ & -0.402826 & -0.309542 & -0.151608 \\
$\mathrm{O}$ & 1.790819 & -0.190290 & -1.053953 \\
$\mathrm{C}$ & 2.736932 & -0.407637 & -0.278897 \\
$\mathrm{~N}$ & 4.004428 & -0.636327 & -0.678895 \\
$\mathrm{C}$ & 4.360449 & -0.668086 & -2.089014 \\
$\mathrm{C}$ & 0.013321 & -2.201714 & 0.444631 \\
$\mathrm{C}$ & 0.375952 & -3.222181 & -0.338734 \\
$\mathrm{P}$ & -0.628139 & 2.033234 & -0.761583 \\
$\mathrm{C}$ & 1.002901 & 2.895803 & -0.758490 \\
$\mathrm{C}$ & -3.074811 & -1.899785 & 1.348833 \\
$\mathrm{C}$ & -1.269435 & 2.472554 & -2.448343 \\
$\mathrm{C}$ & -1.629958 & 3.119619 & 0.347863 \\
$\mathrm{C}$ & 5.067100 & -0.850581 & 0.290892 \\
$\mathrm{Br}$ & 0.652194 & 0.700434 & 2.372453 \\
$\mathrm{H}$ & 2.586096 & -0.404933 & 0.808238 \\
$\mathrm{H}$ & 3.452717 & -0.540224 & -2.678520 \\
$\mathrm{H}$ & 4.827956 & -1.628123 & -2.339904 \\
$\mathrm{H}$ & 5.068434 & 0.136387 & -2.325897 \\
$\mathrm{H}$ & 5.528301 & -1.835723 & 0.146479 \\
$\mathrm{H}$ & 4.656783 & -0.797908 & 1.301937 \\
$\mathrm{H}$ & 5.845633 & -0.084216 & 0.187047 \\
$\mathrm{H}$ & 0.046870 & -2.298106 & 1.532028 \\
$\mathrm{H}$ & 0.361644 & -3.160271 & -1.426518 \\
$\mathrm{H}$ & 0.738769 & -4.164520 & 0.078693 \\
$\mathrm{H}$ & 0.885785 & 3.957153 & -1.006630
\end{tabular}




$\begin{array}{rrrr}\mathrm{H} & 1.439974 & 2.790170 & 0.238405 \\ \mathrm{H} & 1.667043 & 2.417881 & -1.482902 \\ \mathrm{H} & -1.527184 & 4.174036 & 0.066312 \\ \mathrm{H} & -2.688095 & 2.845427 & 0.303623 \\ \mathrm{H} & -1.271957 & 2.973054 & 1.371244 \\ \mathrm{H} & -1.215854 & 3.552508 & -2.629903 \\ \mathrm{H} & -0.671478 & 1.955407 & -3.205915 \\ \mathrm{H} & -2.309910 & 2.151006 & -2.560160 \\ \mathrm{H} & -4.867240 & -0.342828 & -0.455936 \\ \mathrm{H} & -3.862107 & 0.884773 & 0.350347 \\ \mathrm{H} & -3.761776 & 0.674080 & -1.411587 \\ \mathrm{H} & -3.982999 & -2.701132 & -1.393036 \\ \mathrm{H} & -2.839360 & -1.896158 & -2.503532 \\ \mathrm{H} & -2.261999 & -3.185161 & -1.428395 \\ \mathrm{H} & -4.139831 & -2.156399 & 1.312897 \\ \mathrm{H} & -2.487404 & -2.806338 & 1.512091 \\ \mathrm{H} & -2.886298 & -1.217999 & 2.183723\end{array}$

\begin{tabular}{lrrr} 
TSiiriv & \multicolumn{3}{l}{} \\
$\mathrm{SCF}$ & Energy: & -1390.09331518 & \\
$\mathrm{C}$ & -3.296342 & 0.539855 & 1.086604 \\
$\mathrm{P}$ & -2.508292 & -0.859385 & 0.181593 \\
$\mathrm{C}$ & -3.765986 & -1.230351 & -1.116803 \\
$\mathrm{Pd}$ & -0.364440 & -0.476613 & -0.391845 \\
$\mathrm{O}$ & 1.842949 & -0.614629 & -0.811472 \\
$\mathrm{C}$ & 2.638106 & -0.751633 & 0.138895 \\
$\mathrm{~N}$ & 3.951785 & -0.991617 & -0.018827 \\
$\mathrm{C}$ & 4.548875 & -1.121345 & -1.341117 \\
$\mathrm{C}$ & -0.492619 & -2.068526 & -1.601181 \\
$\mathrm{C}$ & -0.966095 & -2.128007 & -2.848613 \\
$\mathrm{P}$ & -0.074352 & 2.265891 & -0.842844 \\
$\mathrm{C}$ & 1.666339 & 2.790966 & -0.471375 \\
$\mathrm{C}$ & -2.669270 & -2.268610 & 1.355977 \\
$\mathrm{C}$ & -0.280366 & 2.954339 & -2.563745 \\
$\mathrm{C}$ & -1.015876 & 3.524103 & 0.149951 \\
$\mathrm{C}$ & 4.830787 & -1.114256 & 1.135030 \\
$\mathrm{Br}$ & 0.161367 & 0.360803 & 2.371017 \\
$\mathrm{H}$ & 2.292150 & -0.665671 & 1.176566 \\
$\mathrm{H}$ & 3.761391 & -1.038392 & -2.089864 \\
$\mathrm{H}$ & 5.044949 & -2.094650 & -1.436154 \\
$\mathrm{H}$ & 5.293927 & -0.332386 & -1.501952 \\
$\mathrm{H}$ & 5.312289 & -2.099772 & 1.147717 \\
$\mathrm{H}$ & 4.251558 & -0.991532 & 2.053036 \\
$\mathrm{H}$ & 5.612481 & -0.345217 & 1.105982 \\
$\mathrm{H}$ & 0.043707 & -2.928347 & -1.187491 \\
$\mathrm{H}$ & -1.507178 & -1.306900 & -3.315334 \\
$\mathrm{H}$ & -0.811588 & -3.011217 & -3.472573 \\
$\mathrm{H}$ & 1.814562 & 3.866177 & -0.629070 \\
$\mathrm{H}$ & 1.883958 & 2.542987 & 0.572148 \\
$\mathrm{H}$ & 2.359165 & 2.235382 & -1.111012 \\
$\mathrm{H}$ & -0.647675 & 4.543107 & -0.022087 \\
$\mathrm{H}$ & -2.079609 & 3.489147 & -0.110223 \\
$\mathrm{H}$ & -0.913461 & 3.275278 & 1.210780 \\
$\mathrm{H}$ & 0.007771 & 4.010988 & -2.631430
\end{tabular}




$\begin{array}{rrrr}\mathrm{H} & 0.333310 & 2.374513 & -3.261684 \\ \mathrm{H} & -1.325472 & 2.854697 & -2.877380 \\ \mathrm{H} & -4.297007 & 0.251917 & 1.430130 \\ \mathrm{H} & -2.665601 & 0.807112 & 1.937634 \\ \mathrm{H} & -3.380703 & 1.406863 & 0.425102 \\ \mathrm{H} & -4.753141 & -1.357324 & -0.657801 \\ \mathrm{H} & -3.810114 & -0.403965 & -1.833254 \\ \mathrm{H} & -3.489925 & -2.137695 & -1.656748 \\ \mathrm{H} & -3.705850 & -2.384359 & 1.693775 \\ \mathrm{H} & -2.340806 & -3.191243 & 0.868311 \\ \mathrm{H} & -2.018419 & -2.071864 & 2.212720\end{array}$

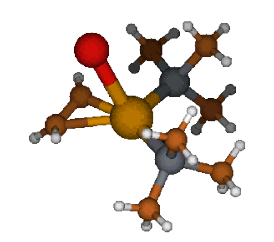

TSi-ii

SCF Energy: -1141.54532462

$\begin{array}{lrrr}\mathrm{C} & 1.563622 & -0.848523 & 1.414549 \\ \mathrm{C} & 1.019904 & -1.785632 & 2.218868 \\ \mathrm{Pd} & -0.092712 & -0.161488 & 0.531580 \\ \mathrm{P} & 0.816234 & 1.974355 & -0.033489 \\ \mathrm{Br} & 1.834102 & -1.758411 & -0.892210 \\ \mathrm{P} & -2.390798 & -0.280620 & -0.149518 \\ \mathrm{H} & 2.474740 & -0.291544 & 1.604849 \\ \mathrm{H} & 0.292395 & -2.508266 & 1.857742 \\ \mathrm{H} & 1.325860 & -1.875645 & 3.261546 \\ \mathrm{C} & 0.923704 & 2.257301 & -1.862171 \\ \mathrm{C} & -0.052823 & 3.514678 & 0.532364 \\ \mathrm{C} & 2.559468 & 2.338144 & 0.479875 \\ \mathrm{C} & -3.528805 & -1.145681 & 1.033496 \\ \mathrm{C} & -3.354219 & 1.268840 & -0.490700 \\ \mathrm{C} & -2.671997 & -1.237682 & -1.710781 \\ \mathrm{H} & -3.737814 & -1.317587 & -1.956335 \\ \mathrm{H} & -2.146820 & -0.748607 & -2.537119 \\ \mathrm{H} & -2.248427 & -2.240571 & -1.600235 \\ \mathrm{H} & -4.385161 & 1.048119 & -0.791933 \\ \mathrm{H} & -3.373367 & 1.894201 & 0.407795 \\ \mathrm{H} & -2.867831 & 1.836736 & -1.290726 \\ \mathrm{H} & -4.553132 & -1.204634 & 0.646020 \\ \mathrm{H} & -3.157663 & -2.158829 & 1.217457 \\ \mathrm{H} & -3.536870 & -0.612158 & 1.989316 \\ \mathrm{H} & 0.433454 & 4.420559 & 0.149946 \\ \mathrm{H} & -1.091594 & 3.498184 & 0.187768 \\ \mathrm{H} & -0.061155 & 3.549709 & 1.626570 \\ \mathrm{H} & 1.406068 & 3.213562 & -2.099107 \\ \mathrm{H} & 1.493008 & 1.437718 & -2.310858 \\ \mathrm{H} & -0.080736 & 2.248791 & -2.297746 \\ \mathrm{H} & 2.893935 & 3.311316 & 0.102092 \\ \mathrm{H} & 2.635926 & 2.337510 & 1.572296 \\ \mathrm{H} & 3.221685 & 1.559601 & 0.087439\end{array}$




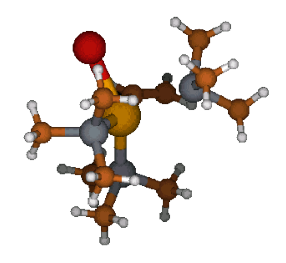

TSii-vi

SCF Energy: -1602.68705693

\begin{tabular}{|c|c|c|c|}
\hline $\mathrm{C}$ & -2.632848 & -2.418738 & 0.911516 \\
\hline $\mathrm{P}$ & -2.467211 & -0.747875 & 0.138638 \\
\hline $\mathrm{C}$ & -3.524898 & 0.253386 & 1.287515 \\
\hline $\mathrm{Pd}$ & -0.328748 & -0.027490 & -0.345977 \\
\hline $\mathrm{P}$ & -0.158934 & 2.050803 & 0.925652 \\
\hline C & 1.555228 & 2.642008 & 1.283532 \\
\hline C & -0.407755 & -1.514708 & -1.741164 \\
\hline C & -0.235921 & -2.836299 & -1.632165 \\
\hline $\mathrm{Br}$ & 1.443522 & 1.107508 & -1.994564 \\
\hline $\mathrm{P}$ & 1.902003 & -1.397886 & 1.082085 \\
\hline C & 3.140312 & -0.640637 & 2.257471 \\
\hline C & 1.593198 & -3.003415 & 1.985256 \\
\hline C & 3.054570 & -1.969538 & -0.253235 \\
\hline $\mathrm{C}$ & -0.869069 & 3.464370 & -0.031976 \\
\hline C & -0.931512 & 2.304405 & 2.598917 \\
\hline $\mathrm{C}$ & -3.582834 & -0.865733 & -1.330245 \\
\hline $\mathrm{H}$ & -0.552637 & -1.086864 & -2.734994 \\
\hline $\mathrm{H}$ & -0.070273 & -3.342653 & -0.682358 \\
\hline $\mathrm{H}$ & -0.214989 & -3.480805 & -2.513628 \\
\hline $\mathrm{H}$ & -4.579036 & -1.219491 & -1.040336 \\
\hline $\mathrm{H}$ & -3.671237 & 0.119720 & -1.798519 \\
\hline $\mathrm{H}$ & -3.147406 & -1.553238 & -2.058580 \\
\hline $\mathrm{H}$ & -4.534818 & -0.169591 & 1.342453 \\
\hline $\mathrm{H}$ & -3.091018 & 0.254729 & 2.291075 \\
\hline $\mathrm{H}$ & -3.596767 & 1.286751 & 0.935101 \\
\hline $\mathrm{H}$ & -3.687613 & -2.679026 & 1.058634 \\
\hline $\mathrm{H}$ & -2.163852 & -3.163737 & 0.265726 \\
\hline $\mathrm{H}$ & -2.122839 & -2.424310 & 1.880137 \\
\hline $\mathrm{H}$ & 1.539002 & 3.677473 & 1.6434 \\
\hline $\mathrm{H}$ & 2.007246 & 2.007076 & 2.05054 \\
\hline $\mathrm{H}$ & 2.151103 & 2.572163 & 0.37066 \\
\hline $\mathrm{H}$ & -0.718216 & 4.419365 & 0.484671 \\
\hline $\mathrm{H}$ & -0.379604 & 3.490795 & -1.00962 \\
\hline $\mathrm{H}$ & -1.941406 & 3.307044 & -0.18936 \\
\hline $\mathrm{H}$ & -0.641185 & 3.276907 & 3.01302 \\
\hline $\mathrm{H}$ & -2.021443 & 2.269300 & 2.53515 \\
\hline $\mathrm{H}$ & -0.600386 & 1.518901 & 3.2865 \\
\hline $\mathrm{H}$ & 3.907000 & -2.530561 & 0.14980 \\
\hline $\mathrm{H}$ & 2.503184 & -2.594267 & -0.96090 \\
\hline $\mathrm{H}$ & 3.409929 & -1.094976 & -0.80658 \\
\hline $\mathrm{H}$ & 2.525590 & -3.532681 & 2.21901 \\
\hline $\mathrm{H}$ & 1.059831 & -2.805350 & 2.92208 \\
\hline $\mathrm{H}$ & 0.965899 & -3.659916 & 1.37366 \\
\hline $\mathrm{H}$ & 3.942832 & -1.343665 & 2.51363 \\
\hline $\mathrm{H}$ & 3.589460 & 0.242900 & 1.7931 \\
\hline $\mathrm{H}$ & 2.644064 & -0.326453 & 3.182 \\
\hline
\end{tabular}




\section{策}

TSiv-V

SCF Energy: -1130.09147263

\begin{tabular}{|c|c|c|c|}
\hline $\mathrm{Sn}$ & 3.144313 & -0.903136 & 0.215833 \\
\hline $\mathrm{C}$ & 1.311374 & -0.914095 & 1.392657 \\
\hline $\mathrm{Pd}$ & -1.014620 & 0.613258 & 0.282035 \\
\hline$P$ & -1.047256 & 2.867081 & 0.049735 \\
\hline $\mathrm{C}$ & -1.720109 & 3.921442 & 1.407250 \\
\hline $\mathrm{H}$ & -1.144679 & 3.764675 & 2.322705 \\
\hline $\mathrm{H}$ & -2.758416 & 3.647422 & 1.613742 \\
\hline $\mathrm{H}$ & -1.675736 & 4.978671 & 1.120867 \\
\hline $\mathrm{C}$ & -2.047371 & 3.404262 & -1.403835 \\
\hline $\mathrm{H}$ & -3.094187 & 3.124294 & -1.250362 \\
\hline $\mathrm{H}$ & -1.979613 & 4.489710 & -1.543486 \\
\hline $\mathrm{H}$ & -1.676533 & 2.886475 & -2.291820 \\
\hline $\mathrm{C}$ & 0.604048 & 3.624825 & -0.259189 \\
\hline $\mathrm{H}$ & 1.248886 & 3.470744 & 0.611138 \\
\hline $\mathrm{H}$ & 1.059694 & 3.132699 & -1.121240 \\
\hline $\mathrm{H}$ & 0.505295 & 4.700072 & -0.450498 \\
\hline O & -2.497835 & -1.294070 & -0.259773 \\
\hline $\mathrm{C}$ & -3.310910 & -1.933610 & 0.407775 \\
\hline $\mathrm{N}$ & -4.130634 & -2.889310 & -0.087206 \\
\hline C & -5.065431 & -3.606704 & 0.761710 \\
\hline $\mathrm{H}$ & -4.985347 & -3.243642 & 1.789920 \\
\hline $\mathrm{H}$ & -4.851399 & -4.682999 & 0.753432 \\
\hline $\mathrm{H}$ & -6.096602 & -3.457388 & 0.417222 \\
\hline C & -4.093886 & -3.236000 & -1.502405 \\
\hline $\mathrm{H}$ & -3.840767 & -4.295998 & -1.626361 \\
\hline $\mathrm{H}$ & -5.071945 & -3.052892 & -1.964039 \\
\hline $\mathrm{H}$ & -3.338139 & -2.620308 & -1.990404 \\
\hline $\mathrm{H}$ & -3.432686 & -1.769225 & 1.491674 \\
\hline $\mathrm{C}$ & -1.928512 & 0.776578 & 2.051264 \\
\hline $\mathrm{C}$ & -1.395803 & 0.856151 & 3.274973 \\
\hline $\mathrm{H}$ & -2.016027 & 0.824430 & 4.173456 \\
\hline $\mathrm{H}$ & -0.324391 & 0.945798 & 3.445559 \\
\hline $\mathrm{H}$ & -3.020276 & 0.717748 & 1.957823 \\
\hline $\mathrm{C}$ & 0.242092 & -1.692550 & 1.133114 \\
\hline $\mathrm{H}$ & -0.584006 & -1.784524 & 1.837184 \\
\hline $\mathrm{H}$ & 0.168807 & -2.296946 & 0.231663 \\
\hline $\mathrm{H}$ & 1.299000 & -0.357059 & 2.331992 \\
\hline $\mathrm{C}$ & 2.959671 & -2.312773 & -1.427062 \\
\hline $\mathrm{H}$ & 2.692160 & -3.308563 & -1.058971 \\
\hline $\mathrm{H}$ & 2.184079 & -1.966077 & -2.114886 \\
\hline $\mathrm{H}$ & 3.907418 & -2.390542 & -1.969795 \\
\hline $\mathrm{C}$ & 4.689329 & -1.592433 & 1.608128 \\
\hline $\mathrm{H}$ & 5.669118 & -1.608936 & 1.118804 \\
\hline $\mathrm{H}$ & 4.756814 & -0.931034 & 2.478551 \\
\hline $\mathrm{H}$ & 4.468716 & -2.603621 & 1.965073 \\
\hline $\mathrm{C}$ & 3.704106 & 1.089200 & -0.457364 \\
\hline $\mathrm{H}$ & 3.683558 & 1.804194 & 0.37184 \\
\hline $\mathrm{H}$ & 4.717040 & 1.076957 & -0.87326 \\
\hline $\mathrm{H}$ & 3.005866 & 1.418308 & -1.230536 \\
\hline $\mathrm{Br}$ & 0.025587 & 0.388578 & -2.122622 \\
\hline
\end{tabular}




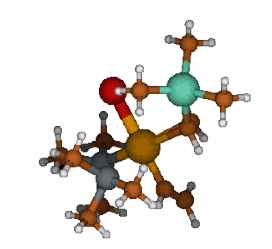

TSVii-V

SCF Energy: -1342.68313472

\begin{tabular}{|c|c|c|c|}
\hline C & -3.531670 & -0.207565 & -1.813513 \\
\hline $\mathrm{P}$ & -3.088214 & -0.871501 & -0.149597 \\
\hline $\mathrm{C}$ & -3.415050 & -2.679219 & -0.323979 \\
\hline $\mathrm{Pd}$ & -0.871911 & -0.473989 & 0.565365 \\
\hline $\mathrm{C}$ & 0.709637 & -1.699180 & 1.624152 \\
\hline C & 1.283409 & -0.454950 & 1.441425 \\
\hline Sn & 2.992989 & -0.104129 & 0.114640 \\
\hline $\mathrm{C}$ & 4.465941 & 0.749697 & 1.494441 \\
\hline C & -4.505793 & -0.296414 & 0.886524 \\
\hline $\mathrm{Br}$ & -0.077828 & -1.386373 & -1.797851 \\
\hline $\mathrm{C}$ & -1.558161 & 0.171275 & 2.341216 \\
\hline C & -1.911970 & -0.521007 & 3.427950 \\
\hline C & 2.712583 & 1.314186 & -1.509065 \\
\hline C & 3.711763 & -2.020202 & -0.618592 \\
\hline $\mathrm{P}$ & -0.967123 & 2.666381 & -0.310499 \\
\hline C & -0.723788 & 3.236584 & -2.065059 \\
\hline C & -2.595407 & 3.500187 & 0.069965 \\
\hline C & 0.175064 & 3.837131 & 0.585689 \\
\hline $\mathrm{H}$ & -4.490222 & 0.795470 & 0.961820 \\
\hline $\mathrm{H}$ & -4.415845 & -0.702896 & 1.896409 \\
\hline $\mathrm{H}$ & -5.458161 & -0.609946 & 0.443954 \\
\hline $\mathrm{H}$ & -2.668612 & -3.103041 & -1.001195 \\
\hline $\mathrm{H}$ & -3.318140 & -3.166205 & 0.651295 \\
\hline $\mathrm{H}$ & -4.421320 & -2.861319 & -0.719078 \\
\hline $\mathrm{H}$ & -3.489120 & 0.885713 & -1.797453 \\
\hline $\mathrm{H}$ & -2.803575 & -0.570857 & -2.542371 \\
\hline $\mathrm{H}$ & -4.541727 & -0.523019 & -2.09941 \\
\hline $\mathrm{H}$ & -1.596705 & 1.264298 & 2.379028 \\
\hline $\mathrm{H}$ & -2.221341 & -0.017487 & 4.345768 \\
\hline $\mathrm{H}$ & -1.908842 & -1.609402 & 3.46785 \\
\hline $\mathrm{H}$ & 0.219442 & -1.970329 & 2.5566 \\
\hline $\mathrm{H}$ & 0.907274 & -2.519539 & 0.937473 \\
\hline $\mathrm{H}$ & 3.600635 & 1.310540 & -2.150327 \\
\hline $\mathrm{H}$ & 2.569892 & 2.331846 & -1.13271 \\
\hline $\mathrm{H}$ & 1.846292 & 1.014464 & -2.102659 \\
\hline $\mathrm{H}$ & 3.923191 & -2.700929 & 0.212387 \\
\hline $\mathrm{H}$ & 2.956180 & -2.472347 & -1.266152 \\
\hline $\mathrm{H}$ & 4.633513 & -1.884134 & -1.19396 \\
\hline $\mathrm{H}$ & 5.400232 & 0.974230 & 0.96868 \\
\hline $\mathrm{H}$ & 4.691735 & 0.052080 & 2.30755 \\
\hline $\mathrm{H}$ & 4.096033 & 1.680620 & 1.93794 \\
\hline $\mathrm{H}$ & 1.178198 & 0.238916 & 2.27787 \\
\hline $\mathrm{H}$ & -0.848773 & 4.321233 & -2.172935 \\
\hline $\mathrm{H}$ & 0.278604 & 2.956602 & -2.40178 \\
\hline $\mathrm{H}$ & -1.442055 & 2.729504 & -2.71787 \\
\hline $\mathrm{H}$ & -0.003151 & 4.885228 & 0.31458 \\
\hline $\mathrm{H}$ & 0.039499 & 3.726890 & 1.66739 \\
\hline $\mathrm{H}$ & 1.215295 & 3.587338 & 0.35394 \\
\hline $\mathrm{H}$ & -2.578680 & 4.572985 & -0.15959 \\
\hline $\mathrm{H}$ & -3.397959 & 3.035504 & -0.513 \\
\hline $\mathrm{H}$ & -2.838648 & 3.374627 & 1.131 \\
\hline
\end{tabular}




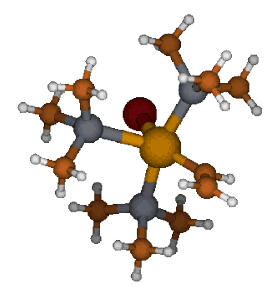

TSVi-vii

SCF Energy: -1602.69495521

\begin{tabular}{|c|c|c|c|}
\hline $\mathrm{C}$ & -3.271963 & 0.519365 & 0.940209 \\
\hline $\mathrm{P}$ & -2.370851 & -0.623028 & -0.194376 \\
\hline C & -3.350754 & -0.494585 & -1.755748 \\
\hline $\mathrm{Pd}$ & -0.039079 & -0.390236 & -0.307791 \\
\hline $\mathrm{P}$ & 2.257072 & -0.862759 & -0.196229 \\
\hline C & -0.120428 & -1.843118 & -1.745531 \\
\hline C & -0.127934 & -1.678187 & -3.073964 \\
\hline $\mathrm{P}$ & 0.153279 & 2.321550 & -0.526315 \\
\hline C & 0.625113 & 3.315342 & 0.967710 \\
\hline $\mathrm{C}$ & -2.836961 & -2.280382 & 0.466483 \\
\hline C & 1.434242 & 2.899656 & -1.751559 \\
\hline C & -1.298760 & 3.315592 & -1.141228 \\
\hline $\mathrm{Br}$ & -0.010348 & -0.137715 & 2.540048 \\
\hline $\mathrm{H}$ & -0.160219 & -2.869220 & -1.363966 \\
\hline $\mathrm{H}$ & -0.089346 & -0.696752 & -3.545179 \\
\hline $\mathrm{H}$ & -0.172266 & -2.529415 & -3.758269 \\
\hline $\mathrm{H}$ & 0.680773 & 4.388736 & 0.747962 \\
\hline $\mathrm{H}$ & -0.103639 & 3.134278 & 1.762938 \\
\hline $\mathrm{H}$ & 1.593296 & 2.972389 & 1.343699 \\
\hline $\mathrm{H}$ & -1.038984 & 4.372467 & -1.278844 \\
\hline $\mathrm{H}$ & -1.647761 & 2.911940 & -2.097920 \\
\hline $\mathrm{H}$ & -2.122715 & 3.254275 & -0.423407 \\
\hline $\mathrm{H}$ & 1.489288 & 3.994080 & -1.806907 \\
\hline $\mathrm{H}$ & 2.420587 & 2.515074 & -1.471340 \\
\hline $\mathrm{H}$ & 1.192237 & 2.506640 & -2.744936 \\
\hline $\mathrm{H}$ & -4.325748 & 0.229171 & 1.0243 \\
\hline $\mathrm{H}$ & -2.789627 & 0.484453 & 1.920510 \\
\hline $\mathrm{H}$ & -3.215182 & 1.543610 & 0.561240 \\
\hline $\mathrm{H}$ & -4.408055 & -0.722602 & -1.578878 \\
\hline $\mathrm{H}$ & -3.268789 & 0.521443 & -2.155867 \\
\hline $\mathrm{H}$ & -2.941495 & -1.186480 & -2.495729 \\
\hline $\mathrm{H}$ & -3.924254 & -2.379348 & 0.563413 \\
\hline $\mathrm{H}$ & -2.462095 & -3.063102 & -0.199643 \\
\hline $\mathrm{H}$ & -2.367478 & -2.400622 & 1.447294 \\
\hline C & 3.287856 & 0.243234 & 0.86381 \\
\hline C & 2.543014 & -2.518361 & 0.562352 \\
\hline C & 3.237412 & -0.938519 & -1.76164 \\
\hline $\mathrm{H}$ & 4.303745 & -0.155661 & 0.96705 \\
\hline $\mathrm{H}$ & 3.345098 & 1.242435 & 0.42079 \\
\hline $\mathrm{H}$ & 2.812456 & 0.320921 & 1.84536 \\
\hline $\mathrm{H}$ & 4.266552 & -1.260437 & -1.56548 \\
\hline $\mathrm{H}$ & 2.758872 & -1.633089 & -2.45617 \\
\hline $\mathrm{H}$ & 3.256623 & 0.050102 & -2.23158 \\
\hline $\mathrm{H}$ & 3.613469 & -2.734247 & 0.65749 \\
\hline $\mathrm{H}$ & 2.073641 & -2.525465 & 1.55039 \\
\hline $\mathrm{H}$ & 2.073333 & -3.291366 & -0.0532 \\
\hline
\end{tabular}




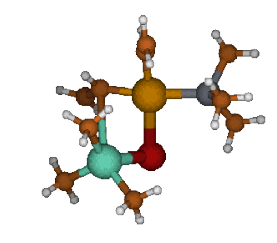

TSV-Viii

SCF Energy: -881.559157096

$\begin{array}{lrrr}\mathrm{C} & 4.470502 & -0.132913 & -0.235932 \\ \mathrm{P} & 2.814840 & -0.715800 & 0.344193 \\ \mathrm{C} & 3.027762 & -0.726355 & 2.181897 \\ \mathrm{Pd} & 0.980950 & 0.594898 & -0.498524 \\ \mathrm{C} & 1.970881 & 2.267308 & -0.016819 \\ \mathrm{C} & 2.140444 & 2.812798 & 1.193402 \\ \mathrm{C} & 2.873434 & -2.518418 & -0.067996 \\ \mathrm{Br} & -0.559809 & -1.568984 & -1.053832 \\ \mathrm{Sn} & -2.180699 & -0.008847 & 0.590448 \\ \mathrm{C} & -3.906891 & 0.064615 & -0.715226 \\ \mathrm{C} & -0.390744 & 1.890098 & -1.319035 \\ \mathrm{C} & -1.039535 & 1.687450 & -2.479794 \\ \mathrm{C} & -2.305348 & -1.605215 & 2.065450 \\ \mathrm{C} & -1.802907 & 1.845611 & 1.662646 \\ \mathrm{H} & 2.396494 & 2.779967 & -0.884408 \\ \mathrm{H} & 1.731395 & 2.377675 & 2.104389 \\ \mathrm{H} & 2.692934 & 3.745269 & 1.326091 \\ \mathrm{H} & 3.769052 & -2.994145 & 0.347884 \\ \mathrm{H} & 1.981381 & -3.015130 & 0.323785 \\ \mathrm{H} & 2.871506 & -2.644902 & -1.155076 \\ \mathrm{H} & 3.932328 & -1.269575 & 2.478728 \\ \mathrm{H} & 3.093830 & 0.304906 & 2.540425 \\ \mathrm{H} & 2.158779 & -1.200571 & 2.649508 \\ \mathrm{H} & 5.282002 & -0.732292 & 0.192280 \\ \mathrm{H} & 4.516670 & -0.195776 & -1.328013 \\ \mathrm{H} & 4.598422 & 0.914121 & 0.052073 \\ \mathrm{H} & -0.513810 & 2.872015 & -0.855453 \\ \mathrm{H} & -1.652466 & 2.465871 & -2.942750 \\ \mathrm{H} & -0.980554 & 0.749493 & -3.029347 \\ \mathrm{H} & -2.361194 & 1.808836 & 2.605648 \\ \mathrm{H} & -0.738460 & 1.964506 & 1.867296 \\ \mathrm{H} & -2.142265 & 2.694785 & 1.066164 \\ \mathrm{H} & -3.026643 & -1.336535 & 2.845815 \\ \mathrm{H} & -2.620441 & -2.537599 & 1.590114 \\ \mathrm{H} & -1.330834 & -1.766654 & 2.535335 \\ \mathrm{H} & -4.789892 & 0.372368 & -0.143854 \\ \mathrm{H} & -3.726051 & 0.783520 & -1.516411 \\ \mathrm{H} & -4.085784 & -0.921545 & -1.150896\end{array}$

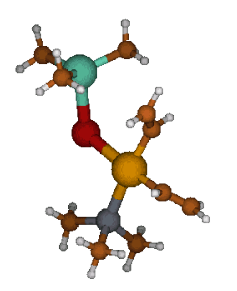

viii

SCF Energy: -881.565580449

$\begin{array}{llrr}\text { C } & -4.282530 & -1.779898 & -0.162511 \\ \text { Sn } & -2.987630 & -0.055510 & 0.075069 \\ \text { C } & -2.857286 & 0.641756 & 2.115472\end{array}$




$\begin{array}{lrrr}\mathrm{Br} & -0.634762 & -1.191170 & -0.413602 \\ \mathrm{Pd} & 1.483911 & 0.641621 & -0.047933 \\ \mathrm{C} & 2.907192 & 2.027630 & 0.169826 \\ \mathrm{C} & 3.791843 & 2.447220 & -0.742456 \\ \mathrm{C} & 0.273026 & 2.261811 & -0.262890 \\ \mathrm{C} & -0.268604 & 3.040262 & 0.687459 \\ \mathrm{P} & 3.099891 & -1.129044 & 0.178438 \\ \mathrm{C} & -3.258094 & 1.425635 & -1.474443 \\ \mathrm{H} & 0.019084 & 2.502510 & -1.303298 \\ \mathrm{C} & 4.273743 & -1.266363 & -1.244446 \\ \mathrm{C} & 2.543946 & -2.884781 & 0.388609 \\ \mathrm{C} & 4.253808 & -0.923175 & 1.609563 \\ \mathrm{H} & 2.936691 & 2.464814 & 1.171810 \\ \mathrm{H} & -0.035461 & 2.915820 & 1.745751 \\ \mathrm{H} & -0.949077 & 3.865136 & 0.457090 \\ \mathrm{H} & -4.013489 & -2.556149 & 0.558872 \\ \mathrm{H} & -4.183973 & -2.189330 & -1.171515 \\ \mathrm{H} & -5.327908 & -1.496216 & 0.000456 \\ \mathrm{H} & -3.118145 & 0.966771 & -2.456718 \\ \mathrm{H} & -2.523542 & 2.220951 & -1.338874 \\ \mathrm{H} & -4.268010 & 1.845669 & -1.418978 \\ \mathrm{H} & -2.595916 & -0.188423 & 2.776980 \\ \mathrm{H} & -3.818677 & 1.059839 & 2.432340 \\ \mathrm{H} & -2.086993 & 1.412837 & 2.176226 \\ \mathrm{H} & 3.793198 & 2.093569 & -1.771986 \\ \mathrm{H} & 4.544108 & 3.200270 & -0.498289 \\ \mathrm{H} & 3.395650 & -3.568315 & 0.484632 \\ \mathrm{H} & 1.916275 & -2.966092 & 1.281509 \\ \mathrm{H} & 1.938310 & -3.185331 & -0.471941 \\ \mathrm{H} & 5.021121 & -1.705825 & 1.628820 \\ \mathrm{H} & 4.734851 & 0.056267 & 1.535089 \\ \mathrm{H} & 3.685659 & -0.954517 & 2.544933 \\ \mathrm{H} & 5.052750 & -2.014452 & -1.057173 \\ \mathrm{H} & 3.721618 & -1.542085 & -2.148734 \\ \mathrm{H} & 4.739506 & -0.290765 & -1.411133 \\ & & & \end{array}$

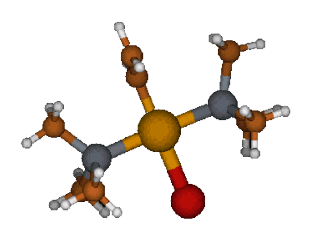

vii

SCF Energy: -1141.61371668

$\begin{array}{lrrr}\mathrm{C} & -0.000077 & 3.250255 & 0.707791 \\ \mathrm{C} & -0.000074 & 2.336467 & -0.270516 \\ \mathrm{Pd} & -0.000008 & 0.330946 & -0.095054 \\ \mathrm{P} & 2.351417 & 0.297681 & -0.068509 \\ \mathrm{Br} & 0.000055 & -2.289014 & 0.143583 \\ \mathrm{P} & -2.351436 & 0.297606 & -0.068521 \\ \mathrm{H} & -0.000134 & 2.691618 & -1.307693 \\ \mathrm{H} & -0.000025 & 2.983699 & 1.764208 \\ \mathrm{H} & -0.000128 & 4.320705 & 0.490909 \\ \mathrm{C} & -3.020194 & -0.385600 & 1.509068 \\ \mathrm{C} & -3.287988 & 1.874763 & -0.270941 \\ \mathrm{C} & -3.094207 & -0.787194 & -1.362201 \\ \mathrm{C} & 3.020319 & -0.386273 & 1.508699 \\ \mathrm{C} & 3.287954 & 1.874938 & -0.270216 \\ \mathrm{C} & 3.094105 & -0.786515 & -1.362746 \\ \mathrm{H} & 4.366740 & 1.698473 & -0.194069\end{array}$




$\begin{array}{rrrr}\text { H } & 3.064452 & 2.313292 & -1.247692 \\ \mathrm{H} & 2.975327 & 2.589945 & 0.494842 \\ \mathrm{H} & 4.113122 & -0.465580 & 1.474596 \\ \mathrm{H} & 2.732638 & 0.265139 & 2.340005 \\ \mathrm{H} & 2.580143 & -1.373346 & 1.673968 \\ \mathrm{H} & 4.181648 & -0.853429 & -1.243571 \\ \mathrm{H} & 2.647421 & -1.780837 & -1.283488 \\ \mathrm{H} & 2.865639 & -0.382629 & -2.354207 \\ \mathrm{H} & -4.366777 & 1.698333 & -0.194740 \\ \mathrm{H} & -2.975376 & 2.590112 & 0.493803 \\ \mathrm{H} & -3.064474 & 2.312681 & -1.248613 \\ \mathrm{H} & -4.113010 & -0.464848 & 1.475150 \\ \mathrm{H} & -2.580072 & -1.372637 & 1.674717 \\ \mathrm{H} & -2.732349 & 0.266157 & 2.340045 \\ \mathrm{H} & -4.181720 & -0.854188 & -1.242807 \\ \mathrm{H} & -2.865969 & -0.383645 & -2.353852 \\ \mathrm{H} & -2.647381 & -1.781427 & -1.282653\end{array}$

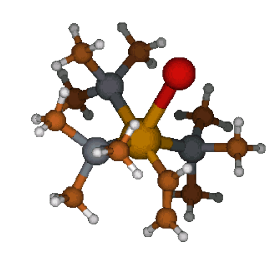

$\mathrm{Vi}$

SCF Energy: -1602.69880496

$\begin{array}{lrrr}\mathrm{C} & -3.622048 & 0.530952 & -0.563773 \\ \mathrm{P} & -2.348280 & -0.793085 & -0.319178 \\ \mathrm{C} & -2.914500 & -1.491176 & 1.291713 \\ \mathrm{C} & -2.929785 & -2.026751 & -1.569163 \\ \mathrm{Pd} & 0.000226 & -0.366122 & -0.317706 \\ \mathrm{P} & 0.001184 & 1.982321 & -1.004000 \\ \mathrm{C} & -1.400157 & 3.054686 & -0.444620 \\ \mathrm{P} & 2.348557 & -0.793540 & -0.317282 \\ \mathrm{C} & 2.930818 & -2.027397 & -1.566716 \\ \mathrm{C} & -0.000284 & -2.305848 & 0.359393 \\ \mathrm{C} & -0.000236 & -3.472552 & -0.295528 \\ \mathrm{C} & 3.622766 & 0.530279 & -0.560892 \\ \mathrm{C} & 2.913369 & -1.491635 & 1.294105 \\ \mathrm{C} & 1.400162 & 3.054836 & -0.439022 \\ \mathrm{C} & 0.004619 & 2.306933 & -2.834680 \\ \mathrm{Br} & -0.002164 & 0.946643 & 2.495957 \\ \mathrm{H} & -0.000637 & -2.311551 & 1.452508 \\ \mathrm{H} & 0.000103 & -3.539417 & -1.383211 \\ \mathrm{H} & -0.000550 & -4.428342 & 0.233568 \\ \mathrm{H} & 1.171661 & 4.103387 & -0.660504 \\ \mathrm{H} & 1.514142 & 2.928028 & 0.641720 \\ \mathrm{H} & 2.331980 & 2.791254 & -0.940056 \\ \mathrm{H} & -1.171017 & 4.103234 & -0.665454 \\ \mathrm{H} & -2.329985 & 2.790788 & -0.949201 \\ \mathrm{H} & -1.518292 & 2.928193 & 0.635712 \\ \mathrm{H} & 0.005060 & 3.381417 & -3.051978 \\ \mathrm{H} & 0.890068 & 1.852995 & -3.291781 \\ \mathrm{H} & -0.879190 & 1.853051 & -3.295014 \\ \mathrm{H} & -4.621870 & 0.084950 & -0.511343 \\ \mathrm{H} & -3.533625 & 1.285386 & 0.220836 \\ \mathrm{H} & -3.507291 & 1.010990 & -1.539988 \\ \mathrm{H} & -3.991262 & -2.257411 & -1.421994 \\ \mathrm{H} & -2.788883 & -1.622162 & -2.576822 \\ \mathrm{H} & -2.344007 & -2.943116 & -1.476231 \\ & & & \end{array}$




$\begin{array}{rrrr}\mathrm{H} & -4.007523 & -1.563068 & 1.318006 \\ \mathrm{H} & -2.482340 & -2.483049 & 1.438032 \\ \mathrm{H} & -2.556641 & -0.836363 & 2.093068 \\ \mathrm{H} & 4.622456 & 0.083945 & -0.508742 \\ \mathrm{H} & 3.508239 & 1.011185 & -1.536712 \\ \mathrm{H} & 3.534530 & 1.284083 & 0.224354 \\ \mathrm{H} & 3.992098 & -2.258351 & -1.418597 \\ \mathrm{H} & 2.344690 & -2.943588 & -1.474275 \\ \mathrm{H} & 2.790924 & -1.622799 & -2.574509 \\ \mathrm{H} & 4.006384 & -1.563212 & 1.321519 \\ \mathrm{H} & 2.554509 & -0.837081 & 2.095220 \\ \mathrm{H} & 2.481351 & -2.483657 & 1.439841\end{array}$

$\mathrm{V}$

SCF Energy: -881.592923383

C

$\mathrm{Sn}$

C

$\mathrm{Br}$

$\mathrm{Pd}$

$\mathrm{Pd}$
$\mathrm{C}$

C

$\mathrm{P}$
$\mathrm{C}$
$\mathrm{C}$

C

C

C

$\mathrm{H}$

$\mathrm{H}$

$\mathrm{H}$

$\mathrm{H}$

$\mathrm{H}$

$\mathrm{H}$

$\mathrm{H}$

$\mathrm{H}$

$\mathrm{H}$

$\mathrm{H}$

$\mathrm{H}$

$\mathrm{H}$

$\mathrm{H}$

$\mathrm{H}$

$\mathrm{H}$

$\mathrm{H}$

$\mathrm{H}$

$\mathrm{H}$

$\mathrm{H}$

$\mathrm{H}$

$\mathrm{H}$

$\mathrm{H}$

$\mathrm{H}$
3.542868

2. 648504

1.844493

$-0.341971$

$-1.033825$

0.632690

1.156166

$-3.061295$

$-1.662153$

$-1.511490$

4.137039

$-3.115014$

$-3.564898$

$-4.544011$

$-2.147619$

1.022126

$-1.846455$

$-1.035411$

0.150303

0.805801

4.340321

3. 974474

2. 781678

4. 950495

3.688345

4.569371

1. 118656

1. 350487

2.648682

$-4.530468$

$-3.638859$

$-2.802513$

$-4.092294$

$-2.337965$

$-2.923945$

$-5.448104$

$-4.411988$

$-4.660079$
$-1.565065$

0.183884

$-0.185267$

$-2.191816$

0.318782

0.568193

1.092854

$-0.011497$

2. 212951

3. 277159

1. 781910

0.796888

$-1.744520$

0.670059

2.348523

2.167661

4. 270818

3. 219046

1. 201633

$-0.463573$

$-1.961979$

$-1.307279$

$-2.336197$

1. 486677

2.699380

2.007820

$-1.000201$

0.712117

$-0.460587$

$-1.753373$

$-2.308354$

$-2.229167$

0.648355

0.366807

1.867063

0.501378

1. 741945

0.178388
$-0.606780$

0.318393

2.303984

$-0.336983$

$-0.561819$

$-2.169393$

$-1.015368$

0.536405

$-0.845421$

$-0.050028$

0.467646

2. 192699

0.896712

$-0.324606$

$-1.818806$

$-0.886094$

$-0.353704$

0.928080

$-2.915260$

$-2.464789$

0.030030

$-1.579126$

$-0.745966$

1. 138905

0.863115

$-0.512471$

2. 254409

2. 691255

2. 994270

1.415736

$-0.036517$

1. 510142

2. 665817

2.831837

2.079926

0.270927

$-0.490403$

$-1.295622$ 


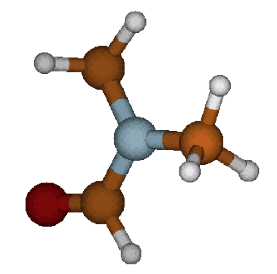

$\mathrm{X} . \mathrm{DMF}$

SCF Energy: -248.502159036

$\begin{array}{lrrr}\mathrm{O} & -0.173953 & -1.917942 & 0.000000 \\ \mathrm{C} & -0.689348 & -0.810285 & 0.000000 \\ \mathrm{~N} & 0.000000 & 0.363488 & 0.000000 \\ \mathrm{H} & -1.787966 & -0.658734 & 0.000000 \\ \mathrm{C} & -0.712043 & 1.637699 & 0.000000 \\ \mathrm{C} & 1.458530 & 0.289059 & 0.000000 \\ \mathrm{H} & 1.871822 & 1.299424 & 0.000000 \\ \mathrm{H} & 1.819105 & -0.247695 & 0.884166 \\ \mathrm{H} & 1.819105 & -0.247695 & -0.884166 \\ \mathrm{H} & 0.014330 & 2.452186 & 0.000000 \\ \mathrm{H} & -1.343804 & 1.751400 & -0.889874 \\ \mathrm{H} & -1.343804 & 1.751400 & 0.889874\end{array}$

xiii.Br

SCF Energy: -13.4719946959

$\begin{array}{llll}\mathrm{Br} & 0.000000 & 0.000000 & 0.000000 \\ \mathrm{Br} & 0.000000 & 0.000000 & 0.000000\end{array}$

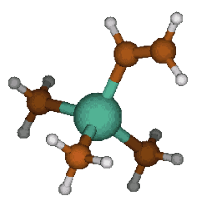

xii.vinilsnMe 3

SCF Energy: -201.097931645

$\begin{array}{lrrr}\mathrm{C} & -1.956620 & 0.126725 & 0.000000 \\ \mathrm{C} & -2.737064 & -0.960142 & 0.000000 \\ \mathrm{H} & -2.451568 & 1.100366 & 0.000000 \\ \mathrm{H} & -3.826422 & -0.897570 & 0.000000 \\ \mathrm{H} & -2.335598 & -1.973637 & 0.000000 \\ \mathrm{Sn} & 0.211631 & 0.089622 & 0.000000 \\ \mathrm{C} & 0.927804 & 2.148719 & 0.000000 \\ \mathrm{C} & 0.927804 & -0.947149 & 1.781449 \\ \mathrm{C} & 0.927804 & -0.947149 & -1.781449 \\ \mathrm{H} & 2.020276 & -1.017435 & 1.773894 \\ \mathrm{H} & 0.622861 & -0.411502 & 2.685691 \\ \mathrm{H} & 0.519012 & -1.961283 & 1.830812 \\ \mathrm{H} & 2.022046 & 2.176310 & 0.000000 \\ \mathrm{H} & 0.573655 & 2.683908 & -0.886841 \\ \mathrm{H} & 0.573655 & 2.683908 & 0.886841 \\ \mathrm{H} & 2.020276 & -1.017435 & -1.773894 \\ \mathrm{H} & 0.519012 & -1.961283 & -1.830812 \\ \mathrm{H} & 0.622861 & -0.411502 & -2.685691\end{array}$




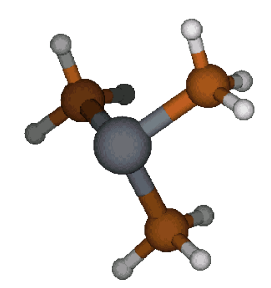

xi.PMe 3

SCF Energy: -461.093900577

$\begin{array}{rrrr}\mathrm{P} & 0.000117 & -0.000015 & -0.605723 \\ \mathrm{C} & -0.888581 & -1.382968 & 0.280701 \\ \mathrm{C} & -0.753711 & 1.460743 & 0.280770 \\ \mathrm{C} & 1.642170 & -0.077898 & 0.280861 \\ \mathrm{H} & -0.231655 & 2.377765 & -0.013783 \\ \mathrm{H} & -1.803065 & 1.568050 & -0.015394 \\ \mathrm{H} & -0.705695 & 1.364826 & 1.372957 \\ \mathrm{H} & -0.460213 & -2.345939 & -0.018367 \\ \mathrm{H} & -0.826042 & -1.294846 & 1.372811 \\ \mathrm{H} & -1.944752 & -1.386326 & -0.010430 \\ \mathrm{H} & 2.256982 & 0.780989 & -0.010109 \\ \mathrm{H} & 1.534652 & -0.078696 & 1.373034 \\ \mathrm{H} & 2.178764 & -0.984869 & -0.018869 \\ & & & \end{array}$

xiv.BrSnMe3

SCF Energy: -136.548424428

$-1.224902$

1.911545

0.000096

$-0.860681$

Sn $\quad-0.677802$

$-0.209871$

$-0.000303$

C $\quad-1.226192$

$-0.000124$

2.085169

1.937685

$-1.701682$

0.000247

C $\quad-1.225408$

$-1.783643$

$-1.223989$

$-2.314819$

$-1.588411$

$-1.297189$

$-0.811132$

$-2.620549$

$-2.229071$

$-0.830418$

2.003605

$-0.783078$

$-2.314165$

2.724005

$-0.923296$

$-0.836235$

2.003828

$-0.804097$

$-0.219287$

$-0.241331$

$-2.315725$

$-1.143751$

$-1.865200$

2.193627

$-0.822915$

$-0.820372$

0.623780

2.485078

2.663978 